\title{
THE GORENSTEINNESS OF THE SYMBOLIC BLOW-UPS FOR CERTAIN SPACE MONOMIAL CURVES
}

\author{
SHIRO GOTO, KOJI NISHIDA, AND YASUHIRO SHIMODA
}

\begin{abstract}
Let $\mathbf{p}=\mathbf{p}\left(n_{1}, n_{2}, n_{3}\right)$ denote the prime ideal in the formal power series ring $A=k[[X, Y, Z]]$ over a field $k$ defining the space monomial curve $X=T^{n_{1}}, Y=T^{n_{2}}$, and $Z=T^{n_{3}}$ with $\operatorname{GCD}\left(n_{1}, n_{2}, n_{3}\right)=1$. Then the symbolic Rees algebras $R_{s}(\mathbf{p})=\bigoplus_{n \geq 0} \mathbf{p}^{(n)}$ are Gorenstein rings for the prime ideals $\mathbf{p}=\mathbf{p}\left(n_{1}, n_{2}, n_{3}\right)$ with $\min \left\{n_{1}, n_{2}, n_{3}\right\}=4$ and $\mathbf{p}=$ $\mathbf{p}(m, m+1, m+4)$ with $m \neq 9,13$. The rings $R_{s}(\mathbf{p})$ for $\mathbf{p}=\mathbf{p}(9,10,13)$ and $\mathbf{p}=\mathbf{p}(13,14,17)$ are Noetherian but non-Cohen-Macaulay, if $\operatorname{ch} k=3$.
\end{abstract}

\section{INTRODUCTION}

Let $k$ be a field and let $A=k[[X, Y, Z]]$ and $S=k[[T]]$ be formal power series rings over $k$. Let $\mathbf{p}=\mathbf{p}\left(n_{1}, n_{2}, n_{3}\right)$ denote, for positive integers $n_{1}, n_{2}$ and $n_{3}$ with $\operatorname{GCD}\left(n_{1}, n_{2}, n_{3}\right)=1$, the kernel of the homomorphism $f: A \rightarrow$ $S$ of $k$-algebras defined by $f(X)=T^{n_{1}}, f(Y)=T^{n_{2}}$, and $f(Z)=T^{n_{3}}$. We put $R_{s}(\mathbf{p})=\sum_{n \geq 0} \mathbf{p}^{(n)} t^{n}$ (here $t$ denotes an indeterminate over $A$ ) and call it the symbolic Rees algebra of $\mathbf{p}$.

In the previous paper [1] the authors studied the problem when $R_{s}(\mathbf{p})$ is a Gorenstein ring and gave a criterion for the case in terms of the elements $f$ and $g$ of $\mathbf{p}$ in Huneke's condition [6] for $R_{s}(\mathbf{p})$ to be Noetherian. With the criterion the authors proved that $R_{s}(\mathbf{p})$ are always Gorenstein for the prime ideals $\mathbf{p}=\mathbf{p}(m, m+1, m+3)$ with $m \geq 1$ and $\mathbf{p}=\mathbf{p}\left(n_{1}, n_{2}, n_{3}\right)$ with $\min \left\{n_{1}, n_{2}, n_{3}\right\}=3$.

To be the next targets we would like to choose the prime ideals $\mathbf{p}=\mathbf{p}(m, m+$ $1, m+4)$ with $m \geq 1$ and $\mathbf{p}=\mathbf{p}\left(n_{1}, n_{2}, n_{3}\right)$ with $\min \left\{n_{1}, n_{2}, n_{3}\right\}=4$, and our conclusion for these ideals can be summarized into the following two theorems.

Theorem (1.1). $R_{s}(\mathbf{p})$ is a Gorenstein ring for $\mathbf{p}=\mathbf{p}(m, m+1, m+4)$, if $m \neq 9,13$.

Theorem (1.2). $R_{s}(\mathbf{p})$ is a Gorenstein ring for $\mathbf{p}=\mathbf{p}\left(n_{1}, n_{2}, n_{3}\right)$, if

$$
\min \left\{n_{1}, n_{2}, n_{3}\right\}=4 \text {. }
$$

In Theorem (1.2) the fact that $R_{s}(\mathbf{p})$ is Noetherian is due to [6]. Our contribution is its Gorensteinness. For $m=9,13$ in Theorem (1.1) the rings $R_{s}(\mathbf{p})$ are Noetherian but not Cohen-Macaulay, if $\operatorname{ch} k=3$ (cf. [7] and (3.4)).

Received by the editors August 15, 1990 and, in revised form, September 10, 1991. 1991 Mathematics Subject Classification. Primary 13H10, 13H15; Secondary 13 E05.

The authors are partially supported by Grant-in-Aid for Cooperative Research. 
Theorem (1.1) (resp. Theorem (1.2)) shall be proved in $\S 3$ (resp. $\S 4$ ). Section 2 is devoted to some preliminary steps. In his remarkable paper [6] Huneke gave a criterion for $R_{s}(\mathbf{p})$ to be Noetherian, by which he guaranteed the Noetherian property of $R_{s}(\mathbf{p})$ for $\mathbf{p}=\mathbf{p}\left(n_{1}, n_{2}, n_{3}\right)$ with $\min \left\{n_{1}, n_{2}, n_{3}\right\}=4$. To prove Theorem (1.2) we need his arguments as well as his results (that we will briefly summarize in $\S 4)$. However the key is the criterion given by the authors [1] for $R_{s}(\mathbf{p})$ to be a Gorenstein ring, which we will recall in $\S 2$ for the sake of completeness.

Throughout this paper let $(A, \mathbf{m})$ be a regular local ring of $\operatorname{dim} A=3$ and p a prime ideal in $A$ with $\operatorname{dim} A / \mathbf{p}=1$. For each finitely generated $A$-module $M$ let $l_{A}(M)$ and $\mu_{A}(M)$ respectively denote the length of $M$ and the number of elements in a minimal system of generators for $M$.

\section{Preliminaries}

First of all let us recall Huneke's criterion.

Proposition (2.1) [6]. If there exist $f \in \mathbf{p}^{(k)}$ and $g \in \mathbf{p}^{(l)}$ with positive integers $k, l$ such that $l_{A}(A /(f, g, x) A)=k l \cdot l_{A}(A / \mathbf{p}+x A)$ for some $x \in \mathbf{m} \backslash \mathbf{p}$, then $R_{s}(\mathbf{p})$ is Noetherian. When the field $A / \mathbf{m}$ is infinite, the converse is also true.

The criterion given by the authors for $R_{S}(\mathbf{p})$ to be a Gorenstein ring is based on (2.1) and is stated as follows.

Theorem (2.2) [1]. Let $f$ and $g$ be as in (2.1). Then the following two conditions are equivalent.

(1) $R_{s}(\mathbf{p})$ is a Gorenstein ring.

(2) $A /(f, g)+\mathbf{p}^{(n)}$ is a Cohen-Macaulay ring for any $1 \leq n \leq k+l-2$.

When this is the case, the A-algebra $R_{s}(\mathbf{p})$ is generated by $\left\{\mathbf{p}^{(n)} t^{n}\right\}_{1 \leq n \leq k+l-2}$, $f t^{k}$ and $g t^{l}$, and the rings $A /(f)+\mathbf{p}^{(n)}, A /(g)+\mathbf{p}^{(n)}$ and $A /(f, g)+\mathbf{p}^{(n)}$ are Cohen-Macaulay for all $n \geq 1$.

Here let us note the following lemma that we will use to calculate the length of certain modules.

Lemma (2.3) ${ }^{1}$. Let $R$ be a two-dimensional Cohen-Macaulay local ring and let $x, y$ be a system of parameters of $R$. For given sequences $p_{0}=0<p_{1} \leq p_{2} \leq$ $\cdots \leq p_{n}$ and $q_{0} \geq q_{1} \geq \cdots \geq q_{n-1}>q_{n}=0$ of integers, let

$$
I=\left(x^{p_{i}} y^{q_{i}} \mid 0 \leq i \leq n\right) R .
$$

Then

$$
l_{R}(R / I)=l_{R}(R /(x, y)) \cdot \sum_{i=1}^{n} q_{i-1}\left(p_{i}-p_{i-1}\right) .
$$

Proof. We may assume that $n \geq 2$ and that our assertion is true for $n-1$. Then considering the sequences $p_{i}^{\prime}=p_{i}(0 \leq i \leq n-1), q_{i}^{\prime}=q_{i}(0 \leq i \leq n-2)$ and $q_{n-1}^{\prime}=0$, we get by the hypothesis on $n$ that

$$
l_{R}\left(R / I^{\prime}\right)=l_{R}(R /(x, y)) \cdot \sum_{i=1}^{n-1} q_{i-1}\left(p_{i}-p_{i-1}\right),
$$

1 The formulation of this lemma is due to the referee. The authors are grateful to the referee for his suggestion. 
where $I^{\prime}=\left(x^{p_{i}^{\prime}} y^{q_{i}^{\prime}} \mid 0 \leq i \leq n-1\right) R$. Since $I^{\prime}=I+\left(x^{p_{n-1}}\right)$ and $I: x^{p_{n-1}}=$ $\left(x^{p_{n}-p_{n-1}}, y^{q_{n-1}}\right)$, we have

$$
\begin{aligned}
l_{R}(R / I) & =l_{R}\left(R / I^{\prime}\right)+l_{R}\left(I+\left(x^{p_{n-1}}\right) / I\right) \\
& =l_{R}(R /(x, y)) \cdot \sum_{i=1}^{n-1} q_{i-1}\left(p_{i}-p_{i-1}\right)+l_{R}\left(R /\left(x^{p_{n}-p_{n-1}}, y^{q_{n-1}}\right)\right) \\
& =l_{R}(R /(x, y)) \cdot \sum_{i=1}^{n} q_{i-1}\left(p_{i}-p_{i-1}\right)
\end{aligned}
$$

as required.

Now let us assume that our ideal $\mathbf{p}$ is generated by the maximal minors of the matrix

$$
M=\left[\begin{array}{lll}
X^{\alpha} & Y^{\beta^{\prime}} & Z^{\gamma^{\prime}} \\
Y^{\beta} & Z^{\gamma} & X^{\alpha^{\prime}}
\end{array}\right]
$$

where $X, Y, Z$ is a regular system of parameters for $A$ and $\alpha, \beta, \gamma, \alpha^{\prime}, \beta^{\prime}, \gamma^{\prime}$ are positive integers. Then after suitable permutations of the rows and columns of $M$, we may assume that the matrix $M$ is one of the following type.

(I) $\alpha \leq \alpha^{\prime}, \beta \leq \beta^{\prime}$ and $\gamma \leq \gamma^{\prime}$,

(II) $\alpha^{\prime}<\alpha, \beta<\beta^{\prime}$ and $\gamma<\gamma^{\prime}$.

As was proved by Herzog and Ulrich [3], p is self-linked (resp. not self-linked) if and only if $M$ has type (I) (resp. type (II)). And in any case it is already known that $\mu_{A}\left(\mathbf{p}^{(2)} / \mathbf{p}^{2}\right)=1$ and $\mathbf{p}^{(n)} \neq \mathbf{p}^{n}$ for all $n \geq 2$ (cf. [5]). However, later we will need so frequently the assertions for the prime ideals $\mathbf{p}$ whose matrices $M$ have type (I) that we would like to give a brief proof for the case. (See [7] for the case of type (II).)

So assume that $\alpha \leq \alpha^{\prime}, \beta \leq \beta^{\prime}$ and $\gamma \leq \gamma^{\prime}$. Let $a=Z^{\gamma+\gamma^{\prime}}-X^{\alpha^{\prime}} Y^{\beta^{\prime}}$, $b=X^{\alpha+\alpha^{\prime}}-Y^{\beta} Z^{\gamma^{\prime}}$ and $c=Y^{\beta+\beta^{\prime}}-X^{\alpha} Z^{\gamma}$. Hence $\mathbf{p}=(a, b, c)$ and any pair of $a, b$ and $c$ forms a regular system of parameters for $A_{\mathbf{p}}$. We begin with the following

Lemma (2.4). $\alpha<\alpha^{\prime}, \beta<\beta^{\prime}$ or $\gamma<\gamma^{\prime}$.

Proof. Suppose that $\alpha=\alpha^{\prime}, \beta=\beta^{\prime}$ and $\gamma=\gamma^{\prime}$. Then since $a-b=$ $\left(X^{\alpha}+Y^{\beta}+Z^{\gamma}\right)\left(Z^{\gamma}-X^{\alpha}\right)$, we have $X^{\alpha}+Y^{\beta}+Z^{\gamma} \in \mathbf{p}$ or $Z^{\gamma}-X^{\alpha} \in \mathbf{p}$, while $\mathbf{p} \subseteq\left(X^{\alpha}, Y^{\beta}, Z^{\gamma}\right)^{2}$. Hence $Z^{\gamma} \in\left(X^{\alpha}, Y^{\beta}, Z^{2 \gamma}\right)$, which is absurd.

Proposition (2.5). There exists $d_{2} \in \mathbf{p}^{(2)}$ such that

$$
\begin{gathered}
X^{\alpha} d_{2}=a c Z^{\gamma^{\prime}-\gamma}-b^{2} Y^{\beta^{\prime}-\beta}, \\
Y^{\beta} d_{2}=a b-c^{2} X^{\alpha^{\prime}-\alpha} Z^{\gamma^{\prime}-\gamma} \text { and } Z^{\gamma} d_{2}=-a^{2}+b c X^{\alpha^{\prime}-\alpha} Y^{\beta^{\prime}-\beta} .
\end{gathered}
$$

If $\alpha<\alpha^{\prime}$, then $d_{2} \equiv-Z^{\gamma+2 \gamma^{\prime}} \bmod (X)$.

Proof. Because $X^{\alpha} a+Y^{\beta^{\prime}} b+Z^{\gamma^{\prime}} c=Y^{\beta} a+Z^{\gamma} b+X^{\alpha^{\prime}} c=0$, we see

$$
\left(X^{\alpha} a+Y^{\beta^{\prime}} b\right) \cdot b=-Z^{\gamma^{\prime}} b c=\left(Y^{\beta} a+X^{\alpha^{\prime}} c\right) \cdot c Z^{\gamma^{\prime}-\gamma}
$$

so that $X^{\alpha}\left(a b-c^{2} X^{\alpha^{\prime}-\alpha} Z^{\gamma^{\prime}-\gamma}\right)=Y^{\beta}\left(a c Z^{\gamma^{\prime}-\gamma}-b^{2} Y^{\beta^{\prime}-\beta}\right)$, whence $X^{\alpha} d_{2}=$ $a c Z^{\gamma^{\prime}-\gamma}-b^{2} Y^{\beta^{\prime}-\beta}$ and $Y^{\beta} d_{2}=a b-c^{2} X^{\alpha^{\prime}-\alpha} Z^{\gamma^{\prime}-\gamma}$ for some $d_{2} \in \mathbf{p}^{(2)}$. Notice 
that

$$
\begin{aligned}
\left(Z^{\gamma} d_{2}\right) b & =\left(Z^{\gamma} b\right) d_{2}=\left(-Y^{\beta} a-X^{\alpha^{\prime}} c\right) d_{2} \\
& =\left(Y^{\beta} d_{2}\right)(-a)+\left(X^{\alpha} d_{2}\right)\left(-c X^{\alpha^{\prime}-\alpha}\right) \\
& =\left(-a^{2}+b c X^{\alpha^{\prime}-\alpha} Y^{\beta^{\prime}-\beta}\right) b
\end{aligned}
$$

and we get $Z^{\gamma} d_{2}=-a^{2}+b c X^{\alpha^{\prime}-\alpha} Y^{\beta^{\prime}-\beta}$, too. If $\alpha<\alpha^{\prime}$, we have $Y^{\beta} d_{2} \equiv$ $a b \equiv-Y^{\beta} Z^{\gamma+2 \gamma^{\prime}} \bmod (X)$ so that $d_{2} \equiv-Z^{\gamma+2 \gamma^{\prime}} \bmod (X)$.

Corollary (2.6) [5]. (1) $\mathbf{p}^{(2)}=\left(d_{2}\right)+\mathbf{p}^{2}$.

(2) $\mu_{A}\left(\mathbf{p}^{(2)}\right) \leq 5$.

(3) $\mathbf{p}^{(n)} \neq \mathbf{p}^{n}$ if $n \geq 2$.

Proof. By (2.4) we may assume that $\alpha<\alpha^{\prime}$. Then as $d_{2} \equiv-Z^{\gamma+2 \gamma^{\prime}} \bmod (X)$ by $(2.5)$ and as $(X)+\mathbf{p}=(X)+\left(Z^{\gamma+\gamma^{\prime}}, Y^{\beta} Z^{\gamma^{\prime}}, Y^{\beta+\beta^{\prime}}\right)$, we have

(\#) $\left(X, d_{2}\right)+\mathbf{p}^{2}=(X)+\left(Z^{\gamma+2 \gamma^{\prime}}, Y^{2 \beta} Z^{2 \gamma^{\prime}}, Y^{\beta+\beta^{\prime}} Z^{\gamma+\gamma^{\prime}}, Y^{2 \beta+\beta^{\prime}} Z^{\gamma^{\prime}}, Y^{2\left(\beta+\beta^{\prime}\right)}\right)$

whence $l_{A}\left(A /\left(X, d_{2}\right)+\mathbf{p}^{2}\right)=3\left(\beta \gamma+\beta \gamma^{\prime}+\beta^{\prime} \gamma^{\prime}\right)$ by (2.3). Let $e_{X A}\left(A / \mathbf{p}^{(2)}\right)$ denote the multiplicity of $A / \mathbf{p}^{(2)}$ relative to the parameter $X$. Then

$$
l_{A}\left(A /(X)+\mathbf{p}^{(2)}\right)=e_{X A}\left(A / \mathbf{p}^{(2)}\right)
$$

since $A / \mathbf{p}^{(2)}$ is a Cohen-Macaulay ring, while we get by the associative formula $[8$, p. 126] of multiplicity that

$$
\begin{aligned}
e_{X A}\left(A / \mathbf{p}^{(2)}\right) & =l_{A_{\mathbf{p}}}\left(A_{\mathbf{p}} / \mathbf{p}^{2} A_{\mathbf{p}}\right) \cdot e_{X A}(A / \mathbf{p})=3 \cdot l_{A}(A /(X)+\mathbf{p}) \\
& =3 \cdot l_{A}\left(A /(X)+\left(Z^{\gamma+\gamma^{\prime}}, Y^{\beta} Z^{\gamma^{\prime}}, Y^{\beta+\beta^{\prime}}\right)\right) \\
& =3\left(\beta \gamma+\beta \gamma^{\prime}+\beta^{\prime} \gamma^{\prime}\right)
\end{aligned}
$$

(cf. (2.3)). Hence $l_{A}\left(A /\left(X, d_{2}\right)+\mathbf{p}^{2}\right)=l_{A}\left(A /(X)+\mathbf{p}^{(2)}\right)$, which yields $(X)+$ $\mathbf{p}^{(2)}=\left(X, d_{2}\right)+\mathbf{p}^{2}$ so that $\mathbf{p}^{(2)}=\left(d_{2}\right)+\mathbf{p}^{2}+X \mathbf{p}^{(2)}$. Thus Nakayama's lemma proves the assertion (1). Notice that $\mu_{A}\left(\mathbf{p}^{(2)}\right)=\mu_{A}\left((X)+\mathbf{p}^{(2)} /(X)\right) \leq 5$ by the above equality (\#) and we have the assertion (2). As $(X)+\mathbf{p}^{2} \subseteq\left(X, Y, Z^{2\left(\gamma+\gamma^{\prime}\right)}\right)$ and as $d_{2} \equiv-Z^{\gamma+2 \gamma^{\prime}} \bmod (X)$, we have $d_{2} \notin(X)+\mathbf{p}^{2}$ so that $d_{2} \notin \mathbf{p}^{2}$; hence $\mathbf{p}^{(2)} \neq \mathbf{p}^{2}$. Let $n \geq 3$ be an integer and assume that $\mathbf{p}^{(n)}=\mathbf{p}^{n}$. Hence $d_{2} t \cdot(a t)^{n-2} \in \mathbf{p}^{n} t^{n-1}$. We put $R=\sum_{i>0} \mathbf{p}^{i} t^{i}$ and $G=R / \mathbf{p} R\left(=\bigoplus_{i \geq 0} \mathbf{p}^{i} / \mathbf{p}^{i+1}\right)$. Then because at is $G$-regular (cf., e.g., [4, 2.1]), we have $d_{2} t \in \mathbf{p} R$, that is $d_{2} \in \mathbf{p}^{2}$ which cannot happen as we have checked above. Thus $\mathbf{p}^{(n)} \neq \mathbf{p}^{n}$ for all $n \geq 2$.

\section{Proof of Theorem (1.1)}

We begin with the following

Theorem (3.1). Suppose that $\mathbf{p}$ is generated by the maximal minors of the matrix

$$
\left[\begin{array}{ccc}
X & Y^{3} & Z^{n+1} \\
Y & Z^{3} & X^{n}
\end{array}\right]
$$

where $X, Y, Z$ is a regular system of parameters for $A$ and $n$ is a positive integer. Then $R_{s}(\mathbf{p})$ is a Gorenstein ring. 
Proof. If $n=1$, then after renaming $X, Y$ and $Z$, we may assume that $\mathbf{p}$ is generated by the maximal minors of the matrix

$$
M=\left[\begin{array}{ccc}
X & Y & Z^{3} \\
Y & Z^{2} & X^{3}
\end{array}\right]
$$

Let us maintain the same notation as in $\S 2$. Then the matrix $M$ is of type (I) and so we have by $(2.5)$ that $d_{2} \equiv-Z^{8} \bmod (X)$. Hence $\left(c, d_{2}, X\right)=\left(X, Y^{2}, Z^{8}\right)$ and

$$
l_{A}\left(A /\left(c, d_{2}, X\right)\right)=16=1 \cdot 2 \cdot l_{A}(A /(X)+\mathbf{p}),
$$

because $l_{A}(A /(X)+\mathbf{p})=l_{A}\left(A /(X)+\left(Z^{5}, Y Z^{3}, Y^{2}\right)\right)=8$ (cf. (2.3)). Thus $R_{s}(\mathbf{p})$ is a Gorenstein ring by $(2.2)$.

Suppose that $n \geq 2$ and recall that $X d_{2}=a c Z^{n-2}-b^{2} Y^{2}$ and $Y d_{2}=$ $a b-c^{2} X^{n-1} Z^{n-2}$ (cf. (2.5)). Then as

$$
\left(X d_{2}+b^{2} Y^{2}\right) b=Z^{n-2} a b c=\left(Y d_{2}+c^{2} X^{n-1} Z^{n-2}\right) c Z^{n-2},
$$

we have $X\left(b d_{2}-c^{3} X^{n-2} Z^{2 n-4}\right)=Y\left(c d_{2} Z^{n-2}-b^{3} Y\right)$ so that

(1) $X d_{3}=c d_{2} Z^{n-2}-b^{3} Y$ and

(2) $Y d_{3}=b d_{2}-c^{3} X^{n-2} Z^{2 n-4}$,

for some $d_{3} \in \mathbf{p}^{(3)}$. When $n=2$, we have $d_{2} \equiv-Z^{9} \bmod (X)$ (cf. (2.5)). Hence as $Y d_{3} \equiv\left(Z^{12}-Y^{11}\right) Y \bmod (X)$ by the equation $(2)$, we get $d_{3} \equiv$ $Z^{12}-Y^{11} \bmod (X)$. Therefore $\left(b, d_{3}, X\right)=\left(X, Y Z^{3}, Z^{12}-Y^{11}\right)$ so that

$$
\begin{aligned}
l_{A}\left(A /\left(b, d_{3}, X\right)\right) & =l_{A}\left(A /\left(X, Y, Z^{12}-Y^{11}\right)\right)+l_{A}\left(A /\left(X, Z^{3}, Z^{12}-Y^{11}\right)\right) \\
& =45=1 \cdot 3 \cdot l_{A}(A /(X)+\mathbf{p}),
\end{aligned}
$$

since $l_{A}(A /(X)+\mathbf{p})=l_{A}\left(A /(X)+\left(Z^{6}, Y Z^{3}, Y^{4}\right)\right)=15$. Thus $R_{s}(\mathbf{p})$ is Noetherian by $(2.1)$. Because $\mathbf{p}^{(2)}=\left(d_{2}\right)+\mathbf{p}^{2}$ (cf. $\left.(2.6)(1)\right)$, we have $(X, b)+\mathbf{p}^{(2)}=$ $\left(X, Z^{9}, Y Z^{3}, Y^{8}\right)$ whence

$$
l_{A}\left(A /(X, b)+\mathbf{p}^{(2)}\right)=30=e_{X A}\left(A /(b)+\mathbf{p}^{(2)}\right),
$$

that is $A /(b)+\mathbf{p}^{(2)}$ is Cohen-Macaulay and so $R_{S}(\mathbf{p})$ is a Gorenstein ring by (2.2).

Now assume that $n \geq 3$. Then since

$$
\left(X d_{3}+b^{3} Y\right) \equiv b c d_{2} Z^{n-2}=\left(Y d_{3}+c^{3} X^{n-2} Z^{2 n-4}\right) c Z^{n-2}
$$

by the equations ( 1$)$ and (2), we have $X\left(b d_{3}-c^{4} X^{n-3} Z^{3 n-6}\right)=Y\left(c d_{3} Z^{n-2}-b^{4}\right)$ so that

(3) $Y d_{4}=b d_{3}-c^{4} X^{n-3} Z^{3 n-6}$

for some $d_{4} \in \mathbf{p}^{(4)}$. Notice that $d_{3} \equiv Z^{3 n+6} \bmod (X)$ by the equation (2) and we get $d_{4} \equiv-Z^{4 n+7}-X^{n-3} Y^{15} Z^{3 n-6} \bmod (X)$ by the equation (3). Hence $\left(c, d_{4}, X\right)=\left(X, Y^{4}, Z^{4 n+7}\right)$ so that

$$
l_{A}\left(A /\left(c, d_{4}, X\right)\right)=4 \cdot(4 n+7)=1 \cdot 4 \cdot l_{A}(A /(X)+\mathbf{p}) .
$$

Thus $R_{s}(\mathbf{p})$ is Noetherian by $(2.1)$. To check that $R_{s}(\mathbf{p})$ is Gorenstein, it is enough by (2.2) to see that $A /(c)+\mathbf{p}^{(2)}$ and $A /(c)+\mathbf{p}^{(3)}$ are Cohen-Macaulay. As $(X, c)+\mathbf{p}^{(2)}=(X)+\left(Z^{2 n+5}, Y^{2} Z^{2 n+2}, Y^{4}\right)$ (cf. (2.6)(1)), we have

$$
l_{A}\left(A /(X, c)+\mathbf{p}^{(2)}\right)=2 \cdot(4 n+7)=e_{X A}\left(A /(c)+\mathbf{p}^{(2)}\right)
$$


whence $A /(c)+\mathbf{p}^{(2)}$ is Cohen-Macaulay. Because $d_{3} \equiv Z^{3 n+6} \bmod (X)$, we have

$$
\begin{aligned}
& \left(X, d_{3}\right)+\mathbf{p p}^{(2)}=(X)+\left(Z^{3 n+6}, Y^{3} Z^{3 n+3}, Y^{4} Z^{2 n+5}, Y^{6} Z^{2 n+2},\right. \\
& \left.Y^{8} Z^{n+4}, Y^{9} Z^{n+1}, Y^{12}\right)
\end{aligned}
$$

by (2.6)(1). Therefore

$$
l_{A}\left(A /\left(X, d_{3}\right)+\mathbf{p p}^{(2)}\right)=6 \cdot(4 n+7)=l_{A}\left(A /(X)+\mathbf{p}^{(3)}\right)
$$

so that $(X)+\mathbf{p}^{(3)}=\left(X, d_{3}\right)+\mathbf{p p}^{(2)}$. Hence

$$
(X, c)+\mathbf{p}^{(3)}=(X)+\left(Z^{3 n+6}, Y^{3} Z^{3 n+3}, Y^{4}\right)
$$

and so we get

$$
l_{A}\left(A /(X, c)+\mathbf{p}^{(3)}\right)=3 \cdot(4 n+7)=e_{X A}\left(A /(c)+\mathbf{p}^{(3)}\right) .
$$

Thus $A /(c)+\mathbf{p}^{(3)}$ is Cohen-Macaulay.

To prove Theorem (1.1) we need one more result.

Proposition (3.2). Suppose that $\mathbf{p}$ is generated by the maximal minors of the matrix

$$
\left[\begin{array}{ccc}
X^{2} & Y^{2} & Z^{3} \\
Y & Z^{2} & X^{2}
\end{array}\right]
$$

where $X, Y, Z$ is a regular system of parameters for $A$. Then $R_{s}(\mathbf{p})$ is a Gorenstein ring.

Proof. The matrix has type (I) and so by (2.5), $Y d_{2}=a b-c^{2} Z$ and $Z^{2} d_{2}=$ $-a^{2}+b c Y$. Therefore as

$$
\left(Y d_{2}+c^{2} Z\right) a=a^{2} b=\left(b c Y-Z^{2} d_{2}\right) b,
$$

we get $Y\left(a d_{2}-b^{2} c\right)=Z\left(-a c^{2}-b d_{2} Z\right)$ so that $Y d_{3}=-a c^{2}-b d_{2} Z$ and $Z d_{3}=a d_{2}-b^{2} c$ for some $d_{3} \in \mathbf{p}^{(3)}$. Notice that

$$
\begin{aligned}
& d_{2} \equiv-Z^{8} \bmod (Y), \quad d_{2} \equiv-X^{6} Y \bmod (Z), \\
& d_{3} \equiv-Z^{12}+X^{10} Z \bmod (Y) \text { and } d_{3} \equiv X^{2} Y^{7} \bmod (Z) .
\end{aligned}
$$

Then we have $c^{2} d_{2}+b d_{3} \equiv 0 \bmod (Z)$, whence $Z d_{4}=c^{2} d_{2}+b d_{3}$ for some $d_{4} \in \mathbf{p}^{(4)}$. Because $d_{4} \equiv X^{14}-2 X^{4} Z^{11} \bmod (Y)$, we see

$$
l_{A}\left(A /\left(d_{2}, d_{4}, Y\right)\right)=l_{A}\left(A /\left(X^{14}, Y, Z^{8}\right)\right)=112=2 \cdot 4 \cdot l_{A}(A /(Y)+\mathbf{p}) .
$$

Thus $R_{S}(\mathbf{p})$ is Noetherian by $(2.1)$. To check that $R_{S}(\mathbf{p})$ is Gorenstein, let $I=\left(d_{2}, d_{3}\right)+\mathbf{p}^{3}\left(\subseteq\left(d_{2}\right)+\mathbf{p}^{(3)}\right)$. Then

$$
(Y)+I=(Y)+\left(Z^{8}, X^{6} Z^{6}, X^{8} Z^{4}, X^{10} Z, X^{12}\right)
$$

so that $l_{A}(A /(Y)+I)=70$ by $(2.3)$, while

$$
\begin{aligned}
e_{Y A}\left(A /\left(d_{2}\right)+\mathbf{p}^{(3)}\right) & =l_{A_{\mathbf{p}}}\left(A_{\mathbf{p}} / d_{2} A_{\mathbf{p}}+\mathbf{p}^{3} A_{\mathbf{p}}\right) \cdot e_{Y A}(A / \mathbf{p}) \\
& =5 \cdot 14=70
\end{aligned}
$$

by the associative formula of multiplicity (cf. $[1,(3.1)(3)]$, too). Hence by the inequalities

$$
l_{A}(A /(Y)+I) \geq l_{A}\left(A /\left(Y, d_{2}\right)+\mathbf{p}^{(3)}\right) \geq e_{Y A}\left(A /\left(d_{2}\right)+\mathbf{p}^{(3)}\right),
$$


we get that $A /\left(d_{2}\right)+\mathbf{p}^{(3)}$ is Cohen-Macaulay. Let $J=\left(d_{2}, d_{4}\right)+d_{3} \mathbf{p}+\mathbf{p}^{4}(\subseteq$ $\left.\left(d_{2}\right)+\mathbf{p}^{(4)}\right)$. Then

$$
(Y)+J=(Y)+\left(Z^{8}, X^{10} Z^{6}, X^{12} Z^{3}, X^{14}\right)
$$

so that $l_{A}(A /(Y)+J)=98=e_{Y A}\left(A /\left(d_{2}\right)+\mathbf{p}^{(4)}\right)$, whence by the inequalities

$$
l_{A}(A /(Y)+J) \geq l_{A}\left(A /\left(Y, d_{2}\right)+\mathbf{p}^{(4)}\right) \geq e_{Y A}\left(A /\left(d_{2}\right)+\mathbf{p}^{(4)}\right),
$$

we find that $A /\left(d_{2}\right)+\mathbf{p}^{(4)}$ is Cohen-Macaulay. Thus $R_{S}(\mathbf{p})$ is a Gorenstein ring by $(2.2)$.

Remark (3.3). The prime ideal $\mathbf{p}=\mathbf{p}(11,14,10)$ corresponds to the ideal considered in (3.2).

Proof of Theorem (1.1). We write $m=4 n+r$ with $0 \leq r<4$. If $r=0$, then $\mathbf{p}=\left(X^{n+1}-Z^{n}, Y^{4}-X^{3} Z\right)$ which is a complete intersection in $A=$ $k[[X, Y, Z]]$. Hence $\mathbf{p}^{(n)}=\mathbf{p}^{n}$ for any $n \geq 1$ and we have an isomorphism $R_{s}(\mathbf{p}) \cong A\left[T_{1}, T_{2}\right] /(f)$ of $A$-algebras, where $A\left[T_{1}, T_{2}\right]$ is a polynomial ring and $0 \neq f \in A\left[T_{1}, T_{2}\right]$. Thus $R_{S}(\mathbf{p})$ is certainly Gorenstein.

(1) $(r=1)$. If $n=0$, then $Y-X^{2} \in \mathbf{p}$ and $\mathbf{p}$ is a complete intersection in $A$. If $n=1$, then $\mathbf{p}=\left(Y^{3}-Z^{2}, X^{3}-Y Z\right)$, which is a complete intersection in $A$. Thus we may assume $n \geq 2$. Then $\mathbf{p}$ is generated by the maximal minors of the matrix

$$
\left[\begin{array}{ccc}
X^{3} & Y^{3} & Z^{n} \\
Y & Z^{2} & X^{n-1}
\end{array}\right]
$$

(cf. [2]), whence the assertion follows from [1, (4.1)] if $n \geq 4$. The cases $n=2,3$ are the exceptional ones, that is $m=9,13$.

(2) $(r=2)$. We may assume $n \geq 1$, because $Z-Y^{2} \in \mathbf{p}$ if $n=0$. Hence p is generated by the maximal minors of the matrix

$$
\left[\begin{array}{ccc}
X^{3} & Y^{2} & Z^{n} \\
Y^{2} & Z & X^{n}
\end{array}\right]
$$

so that the assertion follows from [1, (4.1)] if $n \geq 3$. When $n=1$, notice that $\mathbf{p}$ is generated by the maximal minors of the matrix

$$
\left[\begin{array}{ccc}
Y^{2} & Z & X^{3} \\
Z & X & Y^{2}
\end{array}\right]
$$

and we have $R_{s}(\mathbf{p})$ to be a Gorenstein ring again by [1, (4.1)]. If $n=2, \mathbf{p}$ is generated by the maximal minors of the matrix

so that $R_{s}(\mathbf{p})$ is Gorenstein by (3.2).

$$
\left[\begin{array}{lll}
Y^{2} & Z^{2} & X^{3} \\
Z & X^{2} & Y^{2}
\end{array}\right]
$$

(3) $(r=3)$. We may assume $n \geq 1$, as $Z-X Y \in \mathbf{p}$ if $n=0$. Hence $\mathbf{p}$ is generated by the maximal minors of the matrix

$$
\left[\begin{array}{ccc}
Z & Y^{3} & X^{n+1} \\
Y & X^{3} & Z^{n}
\end{array}\right]
$$

so that the assertion follows from (3.1). This completes the proof of Theorem (1.1).

The symbolic Rees algebras $R_{s}(\mathbf{p})$ for $\mathbf{p}=\mathbf{p}(9,10,13)$ is Noetherian but not Cohen-Macaulay, if ch $k=3$ (cf. [7]). The same is true for $\mathbf{p}=\mathbf{p}(13,14,17)$ too, if $\operatorname{ch} k=3$. We shall prove it in the following 
Example (3.4). Let $\mathbf{p}=\mathbf{p}(13,14,17)$ and let $\mathbf{M}$ denote the unique graded maximal ideal of $R_{s}(\mathbf{p})$. Then $R_{S}(\mathbf{p})$ is a Noetherian ring with $\operatorname{dim} R_{S}(\mathbf{p})_{\mathbf{M}}=4$ and depth $R_{s}(\mathbf{p})_{\mathbf{M}}=3$, if $\operatorname{ch} k=3$.

Proof. The ideal $\mathbf{p}$ is generated by the maximal minors of the matrix

$$
M=\left[\begin{array}{ccc}
X^{3} & Y^{3} & Z^{3} \\
Y & Z & X^{2}
\end{array}\right]
$$

of type (II). Let $a=Z^{4}-X^{2} Y^{3}, b=X^{5}-Y Z^{3}$ and $c=Y^{4}-X^{3} Z$ (hence $\mathbf{p}=(a, b, c))$. Then as $X^{3} a+Y^{3} b+Z^{3} c=Y a+Z b+X^{2} c=0$, we have $Y^{3} a^{3}+Z^{3} b^{3}+X^{6} c^{3}=0$. Therefore because

$$
\left(Z^{3} b^{3}+X^{6} c^{3}\right) b=-Y^{3} a^{3} b=\left(X^{3} a+Z^{3} c\right) a^{3}
$$

we see $X^{3}\left(a^{4}-b c^{3} X^{3}\right)=Z^{3}\left(b^{4}-a^{3} c\right)$ so that $Z^{3} d_{4}=a^{4}-b c^{3} X^{3}$ for some $d_{4} \in \mathbf{p}^{(4)}$. Notice that $c \equiv Y^{4}$ and $d_{1} \equiv Z^{13} \bmod (X)$ and we find

$$
l_{A}\left(A /\left(c, d_{4}, X\right)\right)=52=1 \cdot 4 \cdot l_{A}(A /(X)+\mathbf{p}) \text {, }
$$

whence $R_{s}(\mathbf{p})$ is Noetherian by (2.1) but non-Cohen-Macaulay by (2.2) and [7, (2.4)]. Because depth $R_{s}(\mathbf{p})_{\mathbf{M}} \geq 3$ by [1, (2.1) and (3.7)(3)] and $\operatorname{dim} R_{s}(\mathbf{p})_{\mathbf{M}}=$ 4 , we get depth $R_{s}(\mathbf{p})_{\mathbf{M}}=3$ as required.

\section{Proof of Theorem (1.2)}

Let $\mathbf{p}=\mathbf{p}\left(n_{1}, n_{2}, n_{3}\right)$ with $n_{1}=4$ and assume that $\mathbf{p}$ is not a complete intersection in $A=A[[X, Y, Z]]$. Hence by [2] the ideal $\mathbf{p}$ is generated by maximal minors of a matrix of the following form

$$
\left[\begin{array}{ccc}
X^{\alpha} & Y^{\beta^{\prime}} & Z^{\gamma^{\prime}} \\
Y^{\beta} & Z^{\gamma} & X^{\alpha^{\prime}}
\end{array}\right]
$$

with positive integers $\alpha, \beta, \gamma, \alpha^{\prime}, \beta^{\prime}$ and $\gamma^{\prime}$. Then as $(X)+\mathbf{p}=(X)+$ $\left(Z^{\gamma+\gamma^{\prime}}, Y^{\beta} Z^{\gamma^{\prime}}, Y^{\beta+\beta^{\prime}}\right)$, we have $l_{A}(A /(X)+\mathbf{p})=\beta \gamma+\beta \gamma^{\prime}+\beta^{\prime} \gamma^{\prime}$ (cf. (2.3)), while $e_{X A}(A / \mathbf{p})=4\left(=n_{1}\right)$. Hence $\beta=\gamma^{\prime}=1$ and $\gamma+\beta^{\prime}=3$, as $\beta \gamma+\beta \gamma^{\prime}+$ $\beta^{\prime} \gamma^{\prime}=4$. We may assume $\gamma=1$ and $\beta^{\prime}=2$ so that solving the equations

$$
4\left(\alpha+\alpha^{\prime}\right)=n_{2}+n_{3}, \quad 3 n_{2}=4 \alpha+n_{3}, \quad 2 n_{3}=4 \alpha^{\prime}+2 n_{2},
$$

we get $n_{2}=2 \alpha+\alpha^{\prime}$ and $n_{3}=2 \alpha+3 \alpha^{\prime}$; hence $\alpha^{\prime}$ is odd, as $\operatorname{GCD}\left(4, n_{2}, n_{3}\right)=$ 1. Thus Theorem (1.2) follows from the next more general

Theorem (4.1). Let $\mathbf{p}$ be a prime ideal in a 3-dimensional regular local ring $A$ and assume that $\mathbf{p}$ is generated by the maximal minors of a matrix of the form

$$
\left[\begin{array}{ccc}
X^{q} & Y^{2} & Z \\
Y & Z & X^{p}
\end{array}\right]
$$

where $X, Y, Z$ is a regular system of parameters for $A$ and $p, q$ are positive integers with $p$ odd. Then $R_{s}(\mathbf{p})$ is a Gorenstein ring.

We divide the proof of Theorem (4.1) into a few parts. First we put $a=$ $Z^{2}-X^{p} Y^{2}, b=X^{p+q}-Y Z$ and $c=Y^{3}-X^{q} Z$. Hence $\mathbf{p}=(a, b, c)$ and any pair of $a, b$ and $c$ forms a regular system of parameters for $A_{\mathrm{p}}$. Choose $0 \leq k \in Z$ so that $k p<q \leq(k+1) p$. Then we get by [6, Proof of 3.14] the following 
Lemma 4.2. There exist elements $e_{n} \in \mathbf{p}^{(n)}(1 \leq n \leq k+2)$ and $f \in \mathbf{p}^{(2 k+3)}$ such that

$$
\begin{array}{rlrl}
e_{n} \equiv Y^{2 n+1} \bmod (X) & & (1 \leq n \leq k+1), \\
e_{k+2} & \equiv(-1)^{k} Z^{2 k+3} \bmod (X) & & \text { if } q<(k+1) p, \\
& \equiv Y^{2 k+5}+(-1)^{k} Z^{2 k+3} \bmod (X) & & \text { if } q=(k+1) p, \\
f & \equiv-Z^{4 k+4} \bmod (X) & & \text { if } q-k p<(k+1) p-q, \\
\equiv Y^{4 k+8} \bmod (X) & & \text { if } q-k p>(k+1) p-q>0 .
\end{array}
$$

The Noetherian property of $R_{s}(\mathbf{p})$ now directly follows from (2.1) and (4.2), because

$$
\begin{aligned}
l_{A}\left(A /\left(b, e_{k+2}, X\right)\right) & =l_{A}\left(A /\left(X, Y Z, Y^{2 k+5}+(-1)^{k} Z^{2 k+3}\right)\right) \\
& =1 \cdot(k+2) \cdot 4 \text { if } q=(k+1) p, \\
l_{A}\left(A /\left(e_{k+1}, f, X\right)\right)= & l_{A}\left(A /\left(X, Y^{2 k+3}, Z^{4 k+4}\right)\right) \\
= & (k+1) \cdot(2 k+3) \cdot 4 \text { if } q-k p<(k+1) p-q
\end{aligned}
$$

and

$$
\begin{aligned}
l_{A}\left(A /\left(e_{k+2}, f, X\right)\right) & =l_{A}\left(A /\left(X, Y^{4 k+8}, Z^{2 k+3}\right)\right) \\
& =(k+2) \cdot(2 k+3) \cdot 4 \text { if } q-k p>(k+1) p-q>0
\end{aligned}
$$

(notice that $q-k p \neq(k+1) p-q$, as $p$ is odd).

To see that $R_{S}(\mathbf{p})$ is a Gorenstein ring we need further informations about the ideals $\mathbf{p}^{(n)}$. We begin with the following

Proposition (4.6). $\mathbf{p}^{(n)}=\mathbf{p}^{n}+\sum_{j=1}^{n} e_{j} \mathbf{p}^{n-j}$ for $1 \leq n \leq k+1$.

Proof. Let $I=\mathbf{p}^{n}+\sum_{j=1}^{n} e_{j} \mathbf{p}^{n-j}$ and

$$
\begin{aligned}
J= & (X)+\left(Z^{2 n}, Y Z^{2 n-1}, \ldots, Y^{n-1} Z^{n+1}, Y^{n} Z^{n}\right) \\
& +\left(Y^{n+2} Z^{n-1}, Y^{n+3} Z^{n-2}, \ldots, Y^{2 n} Z, Y^{2 n+1}\right) .
\end{aligned}
$$

Then $(X)+I \supseteq J$, because

$$
\begin{aligned}
a^{n-j} b^{j} & \equiv Y^{j} Z^{2 n-j} \bmod (X) \text { for } 0 \leq j \leq n, \\
b^{n-1-j} e_{j+1} & \equiv Y^{n+2+j} Z^{n-1-j} \bmod (X) \text { for } 0 \leq j \leq n-1 .
\end{aligned}
$$

As $l_{A}(A / J)=4\left(\begin{array}{c}n+1 \\ 2\end{array}\right)=e_{X A}\left(A / \mathbf{p}^{(n)}\right)$ (cf. (2.3)), by the canonical inequalities

$$
l_{A}(A / J) \geq l_{A}(A /(X)+I) \geq l_{A}\left(A /(X)+\mathbf{p}^{(n)}\right) \geq e_{X A}\left(A / \mathbf{p}^{(n)}\right)
$$

we get $J=(X)+I=(X)+\mathbf{p}^{(n)}$. Hence $\mathbf{p}^{(n)}=I+X \mathbf{p}^{(n)}$ so that $\mathbf{p}^{(n)}=I$ by Nakayama's lemma.

Corollary (4.7). $R_{s}(\mathbf{p})$ is a Gorenstein ring, if $q=(k+1) p$.

Proof. By (4.6) and its proof we see $(X, b)+\mathbf{p}^{(n)}=(X)+\left(Z^{2 n}, Y Z, Y^{2 n+1}\right)$ so that

$$
l_{A}\left(A /(X, b)+\mathbf{p}^{(n)}\right)=4 n=e_{X A}\left(A /(b)+\mathbf{p}^{(n)}\right)
$$


for $1 \leq n \leq k+1$. Hence $A /(b)+\mathbf{p}^{(n)}$ is a Cohen-Macaulay ring, which proves by $(2.2)$ and $(4.3)$ the assertion.

Proposition (4.8). Suppose $q<(k+1) p$. Then $\mathbf{p}^{(n)}=\mathbf{p}^{n}+\sum_{j=1}^{k+2} e_{j} \mathbf{p}^{(n-j)}$ for $k+2 \leq n \leq 2 k+2$.

Proof. Let $I=\mathbf{p}^{n}+\sum_{j=1}^{k+2} e_{j} \mathbf{p}^{(n-j)}$ and

$$
\begin{aligned}
J= & (X)+\left(Z^{2 n-1}, Y Z^{2 n-2}, \ldots, Y^{n-k-2} Z^{n+k+1}\right) \\
& +\left(Y^{n-k} Z^{n+k}, Y^{n-k+1} Z^{n+k-1}, \ldots, Y^{n} Z^{n}\right) \\
& +\left(Y^{n+2} Z^{n-1}, Y^{n+3} Z^{n-2}, \ldots, Y^{n+k+2} Z^{n-k-1}\right) \\
& +\left(Y^{n+k+4} Z^{n-k-2}, Y^{n+k+5} Z^{n-k-3}, \ldots, Y^{2 n+2}\right) .
\end{aligned}
$$

Then $(X)+I \supseteq J$, because

$$
\begin{aligned}
& a^{n-k-2-j} b^{j} e_{k+2} \equiv(-1)^{k+j} Y^{j} Z^{2 n-j-1} \bmod (X) \text { for } 0 \leq j \leq n-k-2 \text {, } \\
& a^{k-j} b^{n-k+j} \equiv(-1)^{n-k+j} Y^{n-j+k} Z^{n+k-j} \bmod (X) \text { for } 0 \leq j \leq k \text {, } \\
& b^{n-j-1} e_{j+1} \equiv(-1)^{n-j-1} Y^{n+j+2} Z^{n-j-1} \bmod (X) \text { for } 0 \leq j \leq k \text {, } \\
& b^{n-k-2-j} e_{k+1} e_{j+1} \equiv(-1)^{n-k+j} Y^{n+k+4+j} Z^{n-k-2-j} \bmod (X) \\
& \text { for } 0 \leq j \leq n-k-2 \text {. }
\end{aligned}
$$

Therefore as $l_{A}(A / J)=e_{X A}\left(A / \mathbf{p}^{(n)}\right)$, we get similarly as in the proof of (4.6) that $J=(X)+I=(X)+\mathbf{p}^{(n)}$. Hence $\mathbf{p}^{(n)}=I$.

Proposition (4.9). Suppose that $q-k p<(k+1) p-q$. Then

$$
\mathbf{p}^{(n)}=\mathbf{p}^{n}+f \mathbf{p}^{(n-2 k-3)}+\sum_{j=1}^{k+2} e_{j} \mathbf{p}^{(n-j)}
$$

for $2 k+3 \leq n \leq 3 k+3$.

Proof. We put $I=\mathbf{p}^{n}+f \mathbf{p}^{(n-2 k-3)}+\sum_{j=1}^{k+2} e_{j} \mathbf{p}^{(n-j)}$ and

$$
\begin{aligned}
J= & (X)+\left(Z^{2 n-2}, Y Z^{2 n-3}, \ldots, Y^{n-2 k-3} Z^{n+2 k+1}\right) \\
& +\left(Y^{n-2 k-1} Z^{n+2 k}, Y^{n-2 k} Z^{n+2 k-1}, \ldots, Y^{n-k-2} Z^{n+k+1}\right) \\
& +\left(Y^{n-k} Z^{n+k}, Y^{n-k+1} Z^{n+k-1}, \ldots, Y^{n} Z^{n}\right) \\
& +\left(Y^{n+2} Z^{n-1}, Y^{n+3} Z^{n-2}, \ldots, Y^{n+k+2} Z^{n-k-1}\right) \\
& +\left(Y^{n+k+4} Z^{n-k-2}, Y^{n+k+5} Z^{n-k-3}, \ldots, Y^{n+2 k+4} Z^{n-2 k-2}\right) \\
& +\left(Y^{n+2 k+6} Z^{n-2 k-3}, Y^{n+2 k+7} Z^{n-2 k-4}, \ldots, Y^{2 n+3}\right) .
\end{aligned}
$$

Then $(X)+I \supseteq J$, because

$a^{n-2 k-3-j} b^{j} f \equiv(-1)^{j+1} Y^{j} Z^{2 n-2-j} \bmod (X)$ for $0 \leq j \leq n-2 k-3$,

$a^{k-j-1} b^{n-2 k-1+j} f \equiv(-1)^{n+j} Y^{n-2 k+1+j} Z^{n+2 k-j} \bmod (X)$ for $0 \leq j \leq k-1$,

$a^{k+j} b^{n-k-j} \equiv(-1)^{n-k-j} Y^{n-k-j} Z^{n+k+j} \bmod (X)$ for $0 \leq j \leq k$,

$b^{n-1-j} e_{j+1} \equiv(-1)^{n-1-j} Y^{n+2+j} Z^{n-1-j} \bmod (X)$ for $0 \leq j \leq k$,

$b^{n-k-2-j} e_{k+1} e_{j+1} \equiv(-1)^{n-k-j} Y^{n+k+4+j} Z^{n-k-2-j} \bmod (X)$ for $0 \leq j \leq k$ and

$b^{n-2 k-3-j}\left(e_{k+1}\right)^{2} e_{j+1} \equiv(-1)^{n-1-j} Y^{n+2 k+6+j} Z^{n-2 k-3-j} \bmod (X)$

for $0 \leq j \leq n-2 k-3$. 
Hence we have $J=(X)+I=(X)+\mathbf{p}^{(n)}$ for $2 k+3 \leq n \leq 3 k+3$ by the same reason as in the proof of (4.6). Thus $\mathbf{p}^{(n)}=I$.

Corollary (4.10). $R_{s}(\mathbf{p})$ is a Gorenstein ring, if $q-k p<(k+1) p-q$.

Proof. It suffices to see that $A /\left(e_{k+1}, f\right)+\mathbf{p}^{(n)}$ is a Cohen-Macaulay ring for each $k+2 \leq n \leq 3 k+2$ (cf. (2.2) and (4.4)); that is enough to check $l_{A}\left(A /\left(X, e_{k+1}, f\right)+\mathbf{p}^{(n)}\right) \leq e_{X A}\left(A /\left(e_{k+1}, f\right)+\mathbf{p}^{(n)}\right)$. However, because

$$
e_{X A}\left(A /\left(e_{k+1}, f\right)+\mathbf{p}^{(n)}\right)=4 \cdot l_{A_{\mathbf{p}}}\left(A_{\mathbf{p}} /\left(e_{k+1}, f\right) A_{\mathbf{p}}+\mathbf{p}^{n} A_{\mathbf{p}}\right)
$$

by the associative formula of multiplicity (cf. [8]) and because $e_{k+1}, f$ forms a super regular sequence in $A_{\mathbf{p}}$ (cf. [1, (3.1)(3)]), we can easily compute the exact value of $e_{X A}\left(A /\left(e_{k+1}, f\right)+\mathbf{p}^{(n)}\right)$ in terms of $n$ and $k$, that is

$$
\begin{aligned}
e_{X A} & \left.A /\left(e_{k+1}, f\right)+\mathbf{p}^{(n)}\right) \\
& =2(2 n-k)(k+1) \quad(k+2 \leq n \leq 2 k+2) \\
& =2\left(6 k n-5 k^{2}-11 k-n^{2}+7 n-6\right) \quad(2 k+3 \leq n \leq 3 k+2),
\end{aligned}
$$

while we now explicitly have the ideal $\left(X, e_{k+1}, f\right)+\mathbf{p}^{(n)}$ by $(4.6),(4.8)$ and (4.9) (cf. their proofs, too). Therefore the required inequality $l_{A}\left(A /\left(X, e_{k+1}, f\right)\right.$ $\left.+\mathbf{p}^{(n)}\right) \leq e_{X A}\left(A /\left(e_{k+1}, f\right)+\mathbf{p}^{(n)}\right)$ can be directly checked, which we would like to leave to the readers.

Proposition (4.11). Suppose that $q-k p>(k+1) p-q>0$. Then we have

$$
\mathbf{p}^{(2 k+3)}=\mathbf{p}^{2 k+3}+(f)+\sum_{j=1}^{k+2} e_{j} \mathbf{p}^{(2 k+3-j)} .
$$

$$
\mathbf{p}^{(n)}=\mathbf{p}^{n}+f \mathbf{p}^{(n-2 k-3)}+\sum_{j=1}^{k+2} e_{j} \mathbf{p}^{(n-j)} \quad \text { for } 2 k+4 \leq n \leq 3 k+4 .
$$

Proof. (1) Let $I=\mathbf{p}^{2 k+3}+(f)+\sum_{j=1}^{k+2} e_{j} \mathbf{p}^{(2 k+3-j)}$ and

$$
\begin{aligned}
J= & (X)+\left(Z^{4 k+5}, Y Z^{4 k+4}, \ldots, Y^{k+1} Z^{3 k+4}\right) \\
& +\left(Y^{k+3} Z^{3 k+3}, Y^{k+4} Z^{3 k+2}, \ldots, Y^{2 k+3} Z^{2 k+3}\right) \\
& +\left(Y^{2 k+5} Z^{2 k+2}, Y^{2 k+6} Z^{2 k+1}, \ldots, Y^{3 k+5} Z^{k+2}\right) \\
& +\left(Y^{3 k+7} Z^{k+1}, Y^{3 k+8} Z^{k}, \ldots, Y^{4 k+8}\right) .
\end{aligned}
$$

Then $(X)+I \supseteq J$, because

$$
\begin{aligned}
& a^{k+1-j} b^{j} e_{k+2} \equiv(-1)^{j+k} Y^{j} Z^{4 k+5-j} \bmod (X) \text { for } 0 \leq j \leq k+1, \\
& a^{k-j} b^{k+3+j} \equiv(-1)^{k+1+j} Y^{k+3+j} Z^{3 k+3-j} \bmod (X) \text { for } 0 \leq j \leq k, \\
& b^{2 k+2-j} e_{j+1} \equiv(-1)^{j} Y^{2 k+5+j} Z^{2 k+2-j} \bmod (X) \text { for } 0 \leq j \leq k, \\
& b^{k+1-j} e_{k+1} e_{j+1} \equiv(-1)^{k+1-j} Y^{3 k+7+j} Z^{k+1-j} \bmod (X) \text { for } 0 \leq j \leq k
\end{aligned}
$$

and

$$
f \equiv Y^{4 k+8} \bmod (X) .
$$

As $l_{A}(A / J)=e_{X A}\left(A / \mathbf{p}^{(2 k+3)}\right)$, we get $J=(X)+I=(X)+\mathbf{p}^{(2 k+3)}$ whence $\mathbf{p}^{(2 k+3)}=I$. 
(2) Let $I=\mathbf{p}^{n}+f \mathbf{p}^{(n-2 k-3)}+\sum_{j=1}^{k+2} e_{j} \mathbf{p}^{(n-j)}$ and

$$
\begin{aligned}
J= & (X)+\left(Z^{2 n-2}, Y Z^{2 n-3}, \ldots, Y^{n-2 k-4} Z^{n+2 k+2}\right) \\
& +\left(Y^{n-2 k-2} Z^{n+2 k+1}, Y^{n-2 k-1} Z^{n+2 k}, \ldots, Y^{n-k-2} Z^{n+k+1}\right) \\
& +\left(Y^{n-k} Z^{n+k}, Y^{n-k+1} Z^{n+k-1}, \ldots, Y^{n} Z^{n}\right) \\
& +\left(Y^{n+2} Z^{n-1}, Y^{n+3} Z^{n-2}, \ldots, Y^{n+k+2} Z^{n-k-1}\right) \\
& +\left(Y^{n+k+4} Z^{n-k-2}, Y^{n+k+5} Z^{n-k-3}, \ldots, Y^{n+2 k+5} Z^{n-2 k-3}\right) \\
& +\left(Y^{n+2 k+7} Z^{n-2 k-4}, Y^{n+2 k+8} Z^{n-2 k-5}, \ldots, Y^{2 n+3}\right) .
\end{aligned}
$$

Then $(X)+I \supseteq J$, as

$$
\begin{aligned}
& a^{n-2 k-4-j} b^{j}\left(e_{k+2}\right)^{2} \equiv(-1)^{j} Y^{j} Z^{2 n-2-j} \bmod (X) \quad \text { for } 0 \leq j \leq n-2 k-4, \\
& a^{k-j} b^{n-2 k-2+j} e_{k+2} \equiv(-1)^{n-k+j} Y^{n-2 k-2+j} Z^{n+2 k+1-j} \bmod (X) \text { for } 0 \leq j \leq k, \\
& a^{k-j} b^{n-k+j} \equiv(-1)^{n-k+j} Y^{n-k+j} Z^{n+k-j} \bmod (X) \quad \text { for } 0 \leq j \leq k, \\
& b^{n-1-j} e_{j+1} \equiv(-1)^{n-1-j} Y^{n+2+j} Z^{n-1-j} \bmod (X) \text { for } 0 \leq j \leq k, \\
& b^{n-k-2-j} e_{k+1} e_{j+1} \equiv(-1)^{n-k-j} Y^{n+k+4+j} Z^{n-k-2-j} \bmod (X) \text { for } 0 \leq j \leq k, \\
& b^{n-2 k-3} f \equiv(-1)^{n-1} Y^{n+2 k+5} Z^{n-2 k-3} \bmod (X) \quad \text { and } \quad \text { for } 0 \leq j \leq n-2 k-4 . \\
& b^{n-2 k-4-j} f e_{j+1} \equiv(-1)^{n-j} Y^{n+2 k+7+j} Z^{n-2 k-4-j} \bmod (X)
\end{aligned}
$$

Because $l_{A}(A / J)=e_{X A}\left(A / \mathbf{p}^{(n)}\right)$, we have $J=(X)+I=(X)+\mathbf{p}^{(n)}$, whence $\mathbf{p}^{(n)}=I$.

Corollary (4.12). $R_{s}(\mathbf{p})$ is a Gorenstein ring, if $q-k p>(k+1) p-q>0$.

Proof. By (2.2) and (4.5) we have only to check that $l_{A}\left(A /\left(X, e_{k+2}, f\right)+\mathbf{p}^{(n)}\right) \leq$ $e_{X A}\left(A /\left(e_{k+2}, f\right)+\mathbf{p}^{(n)}\right)$ for $k+3 \leq n \leq 3 k+3$. Because we explicitly know the ideals $\left(X, e_{k+2}, f\right)+\mathbf{p}^{(n)}$ by $(4.6),(4.8)$ and (4.11) and because

$$
\begin{aligned}
e_{X A}( & \left.A /\left(e_{k+2}, f\right)+\mathbf{p}^{(n)}\right) \\
& =4 k n-2 k^{2}+8 n-6 k-4 \quad(k+3 \leq n \leq 2 k+3), \\
& =12 k n-2 n^{2}+18 n-10 k^{2}-26 k-16 \quad(2 k+4 \leq n \leq 3 k+3)
\end{aligned}
$$

we are able to directly check the required inequality. This completes the proof of Theorem (4.1) as well as that of (4.12).

\section{REFERENCES}

1. S. Goto, K. Nishida, and Y. Shimoda, The Gorensteinness of symbolic Rees algebras for space curves, J. Math. Soc. Japan 43 (1991), 465-481.

2. J. Herzog, Generators and relations of abelian semigroups and semigroup rings, Manuscripta Math. 3 (1970), 175-193.

3. J. Herzog and B. Ulrich, Self-linked curve singularities, Nagoya Math. J. 120 (1990), 129153.

4. C. Huneke, On the finite generation of symbolic blow-ups, Math. Z. 179 (1982), 465-472.

5. _ The primary components of and integral closures of ideals in 3-dimensional regular local rings, Math. Ann. 275 (1986), 617-635.

6. __ Hilbert functions and symbolic powers, Michigan Math. J. 34 (1987), 293-318. 
7. M. Morimoto and S. Goto, Non-Cohen-Macaulay symbolic blow-ups for space monomial curves, Proc. Amer. Math. Soc. 116 (1992), 305-311.

8. J. P. Serre, Algèbre locale: multiplicité (3rd ed.), Lecture Notes in Math., vol. 11, SpringerVerlag, 1975.

Department of Mathematics, School of Science and Technology, Meiji University, Higashimita, Tama-Ku, Kawasaki-shi, Japan

Department of Mathematics, School of Science and Technology, Chiba University, YAYOI-CHO, ChIBA-SHI, JAPAN

College of Liberal Arts and Sciences, Kitasato University, Kitasato, Sagamihara-shi, JAPAN 